\title{
\begin{tabular}{l|l} 
MitTraries & DSpace@MIT
\end{tabular}
}

\author{
MIT Open Access Articles
}

\section{Densification and Strain Hardening of a Metallic Glass under Tension at Room Temperature}

The MIT Faculty has made this article openly available. Please share how this access benefits you. Your story matters.

Citation: Wang, Z. T., J. Pan, Y. Li, and C. A. Schuh. “Densification and Strain Hardening of a Metallic Glass under Tension at Room Temperature." Physical Review Letters 111, no. 13 (September 2013). (C) 2013 American Physical Society

As Published: http://dx.doi.org/10.1103/PhysRevLett.111.135504

Publisher: American Physical Society

Persistent URL: http://hdl.handle.net/1721.1/84981

Version: Final published version: final published article, as it appeared in a journal, conference proceedings, or other formally published context

Terms of Use: Article is made available in accordance with the publisher's policy and may be subject to US copyright law. Please refer to the publisher's site for terms of use. 


\title{
Densification and Strain Hardening of a Metallic Glass under Tension at Room Temperature
}

\author{
Z. T. Wang, ${ }^{1}$ J. Pan, ${ }^{1}{ }^{\text {Y. }} \mathrm{Li},{ }^{1,2, *}$ and C. A. Schuh ${ }^{3, \dagger}$ \\ ${ }^{1}$ Department of Materials Science and Engineering, National University of Singapore, Singapore 117576 \\ ${ }^{2}$ Shenyang National Laboratory for Materials Science, Institute of Metal Research, Chinese Academy of Sciences, \\ Shenyang 110016, China \\ ${ }^{3}$ Department of Materials Science and Engineering, Massachusetts Institute of Technology, \\ Cambridge, Massachusetts 02139, USA
}

(Received 9 July 2013; published 26 September 2013)

\begin{abstract}
The deformation of metallic glasses involves two competing processes: a disordering process involving dilatation, free volume accumulation, and softening, and a relaxation process involving diffusional ordering and densification. For metallic glasses at room temperature and under uniaxial loading, disordering usually dominates, and the glass can fail catastrophically as the softening process runs away in a localized mode. Here we demonstrate conditions where the opposite, unexpected, situation occurs: the densifying process dominates, resulting in stable plastic deformation and work hardening at room temperature. We report densification and hardening during deformation in a $\mathrm{Zr}$-based glass under multiaxial loading, in a notched tensile geometry. The effect is driven by stress-enhanced diffusional relaxation, and is attended by a reduction in exothermic heat and hardening signatures similar to those observed in the classical thermal relaxation of glasses. The result is significant, stable, plastic, extensional flow in metallic glasses, which suggest a possibility of designing tough glasses based on their flow properties.
\end{abstract}

DOI: 10.1103/PhysRevLett.111.135504

PACS numbers: $62.20 . \mathrm{F}-$

Although the detailed structure of metallic glasses remains mysterious [1,2], it is understood that there are two opposing processes of structural evolution [3-6] that combine to explain many of their observed properties. One process is associated with structural disordering and is often described as free volume generation, which is accelerated by shear deformation $[7,8]$. The other is a diffusional relaxation or ordering process often referred to as free-volume annihilation [3,4,9]. At low temperatures (including room temperature), the mechanical behavior of metallic glasses $[10,11]$ is dominated by a runaway process of free volume generation, with associated strain softening [12-16], formation of shear bands, and spontaneous failure. This runaway failure process is usually unchecked at low temperatures, where diffusion is slow and thus the rate of free volume annihilation is negligible compared to its rate of accumulation during deformation. Following many hundreds of experimental observations of such runaway softening, it is now regarded as defining of metallic glass deformation at low temperatures.

However, we propose that the opposite situation, where diffusional relaxation can outpace its accumulation at room temperature, although not yet observed, is not formally proscribed by the physics of glass flow. Because tensile mean stresses can greatly enhance diffusion in solids [17-20], it is possible that a high stress state could induce significant diffusion even at room temperature in metallic glasses. Under such conditions the conventional view that free volume only accumulates but is not destroyed during a mechanical deformation may require reevaluation.
At room temperature, what kind and level of stress would be required for significant diffusional free volume annihilation to occur in a metallic glass? For a typical $\mathrm{Zr}$ based metallic glass loaded in uniaxial tension, Fig. 1(a), the rate of free volume increase is large compared to that of diffusional annihilation; the relative rates may be estimated using the widely used free volume evolution model of Spaepen [1,8], as shown in Fig. 1(b), and elaborated on in the Supplemental Material 1 [21]. Over the entire stress range, deformation is associated with net dilatation, which causes softening, and, in turn, final runaway localization and failure [Fig. 1(c)].

However, under somewhat modified stress states that suppress shear flow and favor diffusion, it may be possible to find a regime where annihilation is more rapid than accumulation. For example, the stress state ahead of a notch is one of triaxial net tension, which should accelerate diffusion and thus promote glass relaxation. With modifications the free-volume evolution equations (shown in the Supplemental Material 1 [21]) may be used to estimate the rate of free volume evolution in such a notched sample [Fig. 1(d)]. Our calculations are shown in Fig. 1(e), where the rate of enhanced annihilation and suppressed accumulation leads to a unique condition: there is a hypothetical crossover at a critical stress, $\sigma_{c}$ [Fig. 1(e)], at which the rate of free volume annihilation approaches and surpasses that of free volume generation. This implies that above $\sigma_{c}$ deformation will enhance free volume annihilation more than its production, causing net negative dilatation (shrinkage) - this in turn would lead to hardening rather 
Uniaxial tensile

(a)
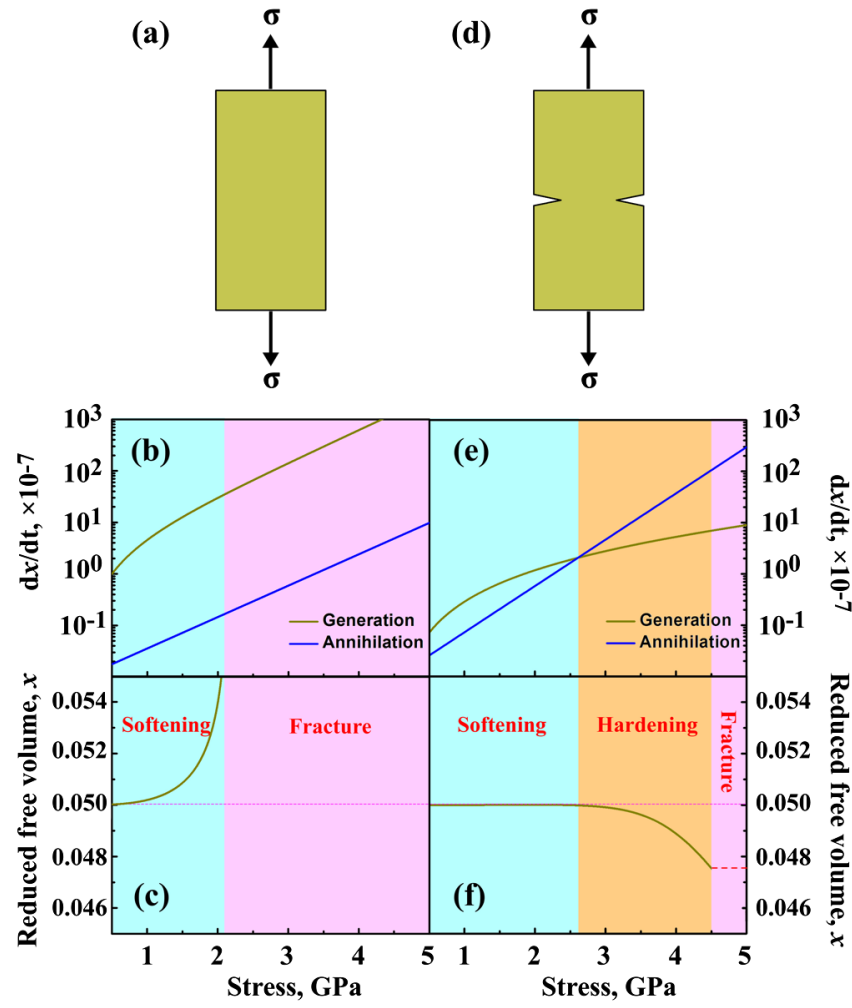

FIG. 1 (color online). The effect of stress state on the rates of free volume generation and annihilation in a metallic glass under tension. Under a uniaxial tensile stress state (a), the rate of generation is always higher than that of annihilation over a wide range of stress (b), resulting in a net increase in free volume and softening. Under a triaxial tensile stress state in a notched sample (d), the rates of generation and annihilation crossover at a critical stress (f) above which the free volume decreases resulting in densification and hardening (e).

than softening [Fig. 1(f)]. With shear banding avoided and a densifying flow induced, the metallic glass should be capable of sustaining genuine extensional plastic deformation beyond the elastic limit.

The experimental alloy with a nominal composition of $\mathrm{Zr}_{64.13} \mathrm{Cu}_{15.75} \mathrm{Ni}_{10.12} \mathrm{Al}_{10}$ (at. \%) was arc-melted under a Ti-gettered argon atmosphere. The ingot was then remelted and tilt casted into a copper mould with cylindrical samples with a diameter of $5 \mathrm{~mm}$ and a length of $75 \mathrm{~mm}$. Notched tensile samples were machined and subsequently subjected to tensile test (Instron 8521 machine) at a constant strain rate $0.02 \mathrm{~mm} / \mathrm{min}$. The morphology of the notched samples was examined by scanning electron microscopy (SEM, Philips XL30 FEG instrument). To characterize the structure of the alloy, cross sections of both as-cast and deformed samples were examined by x-ray diffraction (XRD) employing a Bruker AXS (D8 ADVANCE), and transmission electron microscopy (TEM, JEM, 2010F). Annealing treatment was performed at different temperatures in a vacuum environment. Thermal properties of

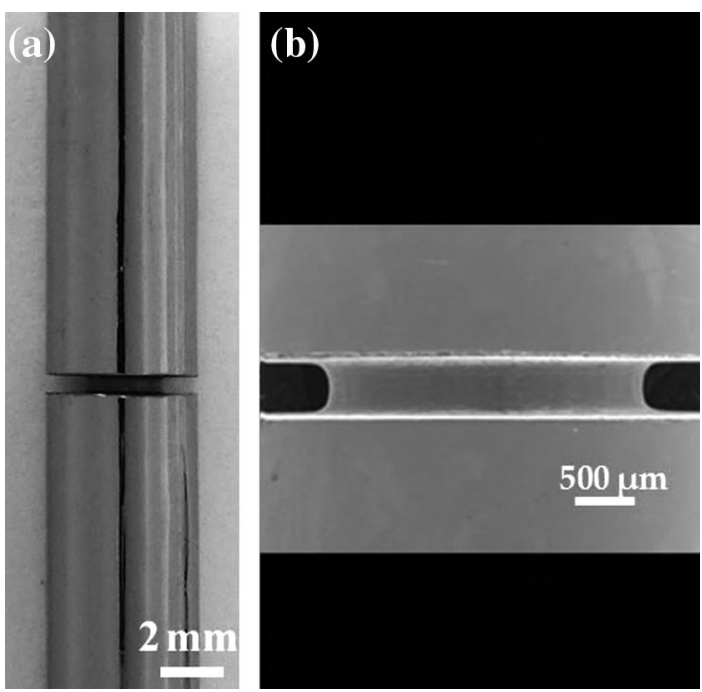

FIG. 2. Optical and SEM micrographs of a deeply notched metallic glass tensile specimen.

as-cast and plastically deformed samples were measured by differential scanning calorimetry (DSC, 2920 TA instruments) at a heating rate of $0.33 \mathrm{~K} \cdot \mathrm{s}^{-1}$. A microhardness tester (Buehler, MicroMet 2103) was used for microhardness measurement.

Figure 2 shows an example of a deeply notched cylindrical test specimen of $\mathrm{Zr}$-based metallic glass, with a thin disk $(\sim 2.4 \mathrm{~mm}$ in diameter and $\sim 0.4 \mathrm{~mm}$ in height) centrally confined. Typical tensile test data are shown in Fig. 3. The data in this plot are presented on axes of characteristic stress vs extension to permit comparison between the notched sample and an unnotched one. The calculated maximum failure stress of such an unconstrained sample is around 1.6 GPa with no plasticity and a maximum $2 \%$ elastic strain. In contrast, our centrally confined samples of low aspect ratio show very different behavior. Their load-displacement curves show considerable elongation (amounting to $\sim 10 \%$ elongation of the neck region) and an apparent strain-hardening behavior similar to that known in many crystalline metals. Yield, i.e., the departure of the curve from linear elasticity, begins at a load higher than that of the unnotched specimen and work hardening occurs progressively to the eventual failure at a load $80 \%$ higher. This plastic flow is stable, as evidenced by the load and unload cycles shown in Fig. 3; in an unloaded condition permanent strain can be seen on the notch region as shown in the inset SEM micrographs. In order to show the changes more clearly, the trace of the notch before deformation is placed on top of the image of the deformed sample. Noticeable elongation (from 394 to $430 \mu \mathrm{m}$, about a $9.1 \%$ increase) and contraction along the horizontal direction (from 2360 to $2258 \mu \mathrm{m}$, about a $4.3 \%$ reduction in area) can be seen. Postinspection by optical and scanning electron microscopy on the cross section of the deformed sample revealed no voids or cracks [inset in 


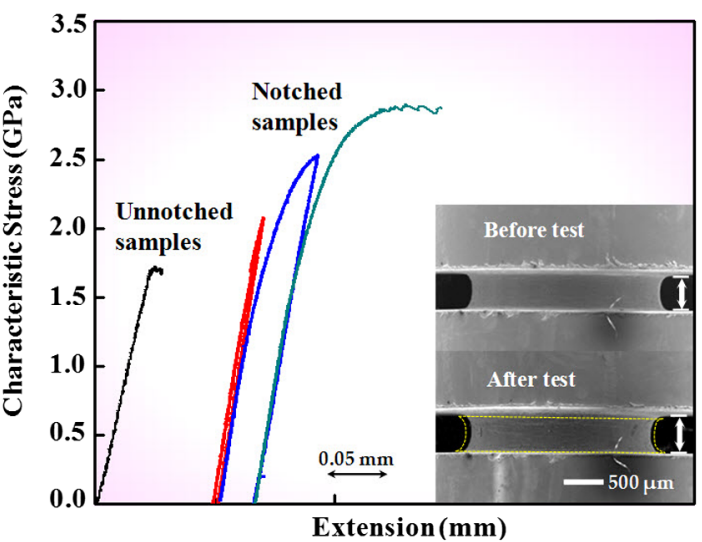

FIG. 3 (color online). Tensile test data for notched and unnotched bars. Here the $y$ axis of the raw load-displacement curves is normalized using the minimum load bearing area of the sample (i.e., the full cross section for an unnotched sample, and the cross section in the notch region for the notched sample), to permit comparison between the two different geometries. The notched specimen exhibits work hardening characteristics and large, stable extensional flow, whereas the unnotched sample fails catastrophically at the elastic limit. The inset shows the permanent extension in the notched region before and after tensile testing.

Fig. 4(a)]. The high plastic elongation is not a product of profuse shear banding either, as only a few small shear traces are observed on the surface of the sample. Furthermore, a TEM investigation (see the Supplemental Material 2 [22].) of the deformed region found no observable changes in structure, effectively ruling out crystallization as the cause for the apparent hardening in our specimen.

As suggested in connection with Fig. 1, we speculate that the strain hardening observed in our samples of Fig. 3 is due to densification, made possible by the multiaxial stress state of the notched tensile samples which geometrically suppresses shear failure and promotes diffusional relaxation (free volume annihilation). If so, the mechanical properties of the metallic glass after deformation should be different, reflective of a relaxed structure. To verify this, microhardness tests were carried out on the deformed specimen after sectioning it longitudinally. Figure 4(a) shows the hardness profiles for the as-cast specimen, and two specimens strained to $2 \%$ and $7 \%$ plastic elongation, measured on lines crossing the notch region. The hardness of the as-cast sample is constant around $3.9 \mathrm{GPa}$ across the notch, while that of the deformed specimens shows a clear rise across the notch with a peak at the center. These data provide clear evidence that the apparent hardening observed during the test in Fig. 3 is in fact a reflection of true hardening in the notch region (and slightly beyond its edges as well).

That plastic strain can lead to relaxation or hardening of a glass is consistent with the theoretical considerations of Fig. 1, and it begs the question of how similar this effect is
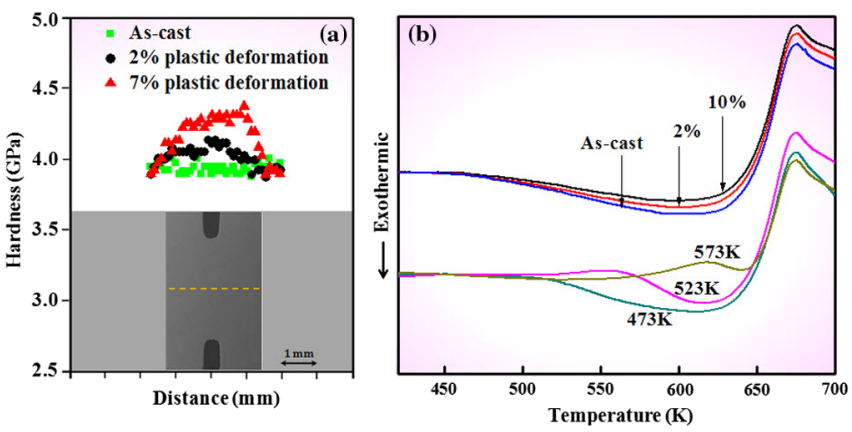

FIG. 4 (color online). Investigation of property changes after tensile deformation. Hardening behavior of the notched region after tensile deformation (a), was revealed by microhardness traces. The DSC curves of the deformed specimen also show a signature reduction in endothermic heat, similar to those specimens after annealing (b), reflective of ordering or densification.

to conventional thermal relaxation. As a first point of comparison, we note that upon annealing at $573 \mathrm{~K}$ for $0.5 \mathrm{~h}$, the present $\mathrm{Zr}$-based glass hardens by about $10 \%$, which is a close match to what is seen in Fig. 4 for the 7\% elongated sample. As a more rigorous comparison, we can assess the free volume changes by examining the endothermic signals shown in differential scanning calorimetry (DSC) curves before the glass transition [9,23,24]. Specimens at various plastic elongation levels were sectioned to remove just the disk of the notch region, which was then subjected to DSC. The results, as shown in Fig. 4(b), reveal a reduction in exothermic heat when compared with the as-cast sample, strikingly similar to what is observed in samples subjected to heat treatment. The values of the exothermic heat before the glass transition for the as-cast, $2 \%$ and $10 \%$ samples are 7.1, 5.9, and $4.4 \mathrm{~J} / \mathrm{g}$, respectively, which correspond to an absolute free volume reduction of $0.016 \%$ and $0.040 \%$, respectively, based on the method in Ref. [23]. This further confirms that the deformation observed in the notched samples experienced structural ordering or free volume annihilation due to plastic deformation.

The notion that deformation can lead to densification is widely reported in many amorphous materials [25-27]. For example, under pressure or indentation amorphous silica can be densified by more than $25 \%$ from its original volume [25]. Polymeric materials also can be densified, even under uniaxial tensile deformation [27]. However, we are not aware that mechanical densification has been previously reported in monolithic metallic glasses, especially in states of net tension, where the normal mode of deformation is one of rapid shear-induced softening and localization. Interestingly, the present observations are somewhat different even from prior work on notched tension testing of other metallic glasses, such as in the work of Flores and Dauskardt [28], where similarly notched specimens exhibited lower strengths and no strain hardening as 
we see in Fig. 3. Their experiments also involved about the same level of triaxiality, i.e., a ratio of mean to deviatoric stress of 1.72 based on the Bridgman method in the present case, similar to the highest value of 2.13 reported by Flores and Daukardt [28]. We believe that the ability to reach the densifying regime as in Figs. 1(d)-1(f) is certainly dependent upon the properties of the individual glass used in the experiments. For example, similar experiments we have conducted on La-based BMGs exhibit responses more like those of Flores and Dauskardt (i.e., no stable plastic extension prior to fracture), and both glasses in those studies are of lower intrinsic toughness $\left(17.4\right.$ and $\left.<2 \mathrm{MPa} \cdot \mathrm{m}^{1 / 2}\right)$ $[29,30]$ than the present glass. The two glasses also likely have different fictive temperatures, which is now known to influence mechanical properties as well [31]. We suggest that it may be the ability of a given glass to achieve the densifying regime under the stress state ahead of a crack tip that is in fact the controlling factor behind the property of fracture toughness.

It should also be noted that the present plastic deformation appears quite homogeneous, and although it is mechanistically like conventional high-temperature homogeneous flow (or creep) of glass $[13,32,33]$, it is different in that it is a densifying flow. Previous reports of homogeneous deformation generally evoke a disordering and free volume raising process $[12,13]$, or a steadystate flow where the two processes of disordering and relaxation are in balance. The present result invokes a crossover to densification-dominated flow that is a result of the very high applied tensile mean stress [Fig. 1(e)].

Our results on bulk-sized samples are essentially based on suppressing shear failure through geometric constraint, leading to the emergence of work hardening. Similar effects may emerge by suppressing shear failure in other ways. For example, it has been proposed that small specimens, below a scale at which shear bands can form, exhibit homogeneous flow; previous reports of homogeneous flow and strain hardening in nanosized samples under tension by Jang et al. [34] and Tian et al. [35] may thus be consistent with our current analysis. Given the geometric constraints around crack tips, fracture and fatigue phenomena in some situations may also be reinterpreted with an eye towards the possibility of local hardening such as we see here. Although inducing higher hardness around a crack tip in principle should correlate with lower local resistance to crack propagation, the local process of hardening is a dissipative mechanism that promotes toughness. The balance between the need for hardening and avoidance of local embrittlement is a topic worthy of greater study. As noted above, we view the possibility of densifying flow around tensile stress concentrations as potentially controlling for fracture properties - the ability to access this mode of deformation may hold the answer to why some glasses are tough and others are brittle. However, the type of hardening observed here is only expected in states of net tensile loading; based on Eq. 5 [21] compressive stresses are expected to suppress diffusion, even though they do promote densification.

C. A.S. acknowledges the support of the U.S. DTRA under Grant No. HDTRA-11-1-0062. Y.L. thanks for financial support from IMR, CAS.

*Corresponding author.

mseliy@nus.edu.sg

${ }^{\dagger}$ Corresponding author. schuh@MIT.EDU

[1] W. Klement, R.H. Willens, and P. Duwez, Nature (London) 187, 869 (1960).

[2] H. W. Sheng, W. K. Luo, F. M. Alamgir, J. M. Bai, and E. Ma, Nature (London) 439, 419 (2006).

[3] F. Spaepen, Acta Metall. 25, 407 (1977).

[4] M. Heggen, F. Spaepenm, and M. Feuerbacher, J. Appl. Phys. 97, 033506 (2005).

[5] C. A. Schuh, T. C. Hufnagel, and U. Ramamurty, Acta Mater. 55, 4067 (2007).

[6] C. A. Pampillo, J. Mater. Sci. 10, 1194 (1975).

[7] A. S. Argon, Acta Metall. 27, 47 (1979).

[8] P. S. Steif, F. Spaepen, and J. W. Hutchinson, Acta Metall. 30, 447 (1982).

[9] A. Van den Beukel and J. Sietsma, Acta Metall. Mater. 38, 383 (1990).

[10] C. A. Schuh and A. C. Lund, Nat. Mater. 2, 449 (2003).

[11] A. Furukawa and H. Tanaka, Nat. Mater. 8, 601 (2009).

[12] F. Spaepen, Nat. Mater. 5, 7 (2006).

[13] R. T. Ott, M. J. Kramer, M. F. Besser, and D. J. Sordelet, Acta Mater. 54, 2463 (2006).

[14] P. De Hey, J. Sietsma, and A. Van den Beukel, Acta Mater. 46, 5873 (1998).

[15] H. Bei, S. Xie, and E. P. George, Phys. Rev. Lett. 96, 105503 (2006).

[16] J. Pan, Q. Chen, L. Liu, and Y. Li, Acta Mater. 59, 5146 (2011).

[17] G. Ruitenberg, P. De Hey, F. Sommer, and J. Sietsma, Phys. Rev. Lett. 79, 4830 (1997).

[18] H. Mehrer, Diffusion in Solids (Springer, New York, 2007), Chap. 8.

[19] F. Faupel, W. Frank, M. P. Macht, H. Mehrer, V. Naundorf, K. Ratzke, H. R. Schober, S. K. Sharma, and H. Teichler, Rev. Mod. Phys. 75, 237 (2003).

[20] M. J. Aziz, P. C. Sabin, and G. Q. Lu, Phys. Rev. B 44, 9812 (1991).

[21] See Supplemental Material 1 at http://link.aps.org/ supplemental/10.1103/PhysRevLett.111.135504 for theoretical analysis of change in free volume upon tensile loading.

[22] See Supplemental Material 2 at http://link.aps.org/ supplemental/10.1103/PhysRevLett.111.135504 for high resolution TEM images.

[23] A. Slipenyuk and J. Eckert, Scr. Mater. 50, 39 (2004).

[24] O. Haruyama, Y. Nakayamaa, R. Wada, H. Tokunaga, J. Okada, T. Ishikawa, and Y. Yokoyama, Acta Mater. 58, 1829 (2010).

[25] P. W. Bridgman and I. Simon, J. Appl. Phys. 24, 405 (1953). 
[26] T. Rouxel, H. Ji, T. Hammouda, and A. Moréac, Phys. Rev. Lett. 100, 225501 (2008).

[27] A. D. Drozdov, J. Appl. Polym. Sci. 74, 1705 (1999).

[28] K. M. Flores and H. R. Dauskardt, Acta Mater. 49, 2527 (2001).

[29] P. Lowhaphandu and J. J. Lewandowsk, Scr. Mater. 38, 1811 (1998).

[30] N. Nagendra, U. Ramamurty, T. T. Goh, and Y. Li, Acta Mater. 48, 2603 (2000).
[31] G. Kumar, P. Neibecker, Y.H. Liu, and J. Schroers, Nat. Commun. 4, 1536 (2013)

[32] J. Lu, G. Ravichandran, and W. L. Johnson, Acta Mater. 51, 3429 (2003).

[33] J.C. Gibeling and W.D. Nix, Scr. Metall. 12, 919 (1978).

[34] D. Jang and J. R. Greer, Nat. Mater. 9, 215 (2010).

[35] L. Tian, Y. Q. Cheng, Z. W. Shan, J. Li, C. C. Wang, X. D. Han, J. Sun, and E. Ma, Nat. Commun. 3, 609 (2012). 